TITLE:

\title{
Preparation for a neutronics experiment using a discharge fusion device and an imaging plate neutron detector
}

\author{
$\operatorname{AUTHOR}(\mathrm{S})$ :
}

Mukai, Keisuke; Konishi, Satoshi

\section{CITATION:}

Mukai, Keisuke ...[et al]. Preparation for a neutronics experiment using a discharge fusion device and an imaging plate neutron detector. Fusion Engineering and Design 2019, 146(Part B): 1633-1636

ISSUE DATE:

2019-09

URL:

http://hdl.handle.net/2433/244111

\section{RIGHT:}

(c) 2019. This manuscript version is made available under the CC-BY-NC-ND 4.0 license

http://creativecommons.org/licenses/by-nc-nd/4.0/; The full-text file will be made open to the public on 1 September 2021 in accordance with publisher's 'Terms and Conditions for Self-Archiving'; この論文は出版社版でありません。引用 の際には出版社版をご確認ご利用ください。; This is not the published version. Please cite only the published version. 


\title{
Preparation for a neutronics experiment using a discharge fusion device and an imaging plate neutron detector
}

\author{
Keisuke Mukai $^{\mathrm{a}}$, Satoshi Konishi ${ }^{\mathrm{a}}$ \\ a Institute of Advanced Energy, Kyoto University, Uji, Kyoto, 611-0011, Japan
}

\begin{abstract}
Tritium breeding ratio (TBR) is one of the most important parameters determining the tritium self-sufficiency of a deuterium-tritium fusion reactor. A neutronics experiment is planned to measure the spectral neutron fluences two-dimensionally (2D) using a discharge-type compact fusion neutron source and a neutron imaging plate (NIP), respectively. We report a calibration method for the NIP and optimization of the discharge condition to enhance the neutron production rate. A linear relationship between the neutron fluence in the NIP and photostimulated luminescence (PSL) per area was obtained in the range of the neutron fluence from $10^{3}$ to $10^{7} \mathrm{n} / \mathrm{cm}^{2}$. A neutron production rate higher than $10^{7} \mathrm{n} / \mathrm{s}$ was successfully achieved by the optimized discharge condition. It is shown that a quantitative 2D measurement by the NIP is feasible using the linear relationship and a correlation coefficient on the energy spectrum.
\end{abstract}

Keywords: neutron measurement, fusion neutron generation, neutron imaging plate, blanket, neutronics.

\section{Introduction}

A tritium breeding ratio (TBR) larger than unity is required for the self-sufficient fueling of a deuteriumtritium (DT) fusion reactor. Up to present, many simulation studies for fusion blankets have been carried out to evaluate the tritium breeding performances with Monte Carlo simulation codes. However, it should be emphasized that an experimental examination of tritium breeding performance with spectral neutron fluxes is vital. This is because errors can be caused by uncertainties in the nuclear data, a discrepancy in the simulated and the actual geometries, and an unexpected neutron absorption by impurities in the blanket materials [1]-[4]. Therefore, it is planned to perform a neutronics experiment with a blanket mock-up initially with a cylindrical discharge-type deuterium-deuterium (DD) fusion device [5],[6], and later with a DT neutron source.

A neutron imaging plate (NIP) with a Gd converter, which has a high spatial resolution $(<200 \mu \mathrm{m})$ and a wide dynamic range $\left(1: 10^{5}\right)$ [7], is used as a twodimensional (2D) neutron detector. The NIP is able to detect an ultra-low neutron fluence, even at the order of $10^{3} \mathrm{n} / \mathrm{cm}^{2}$ [8], which is as good as a single neutron (s.n.) per pixel of $200 \mu \mathrm{m}$ square. However, a neutron fluence ten times greater than the limit is required for a clear neutron radiograph and quantitative analysis as a low neutron fluence generates a high statistical error [8]. In the previous work, the maximum neutron yield of the device was less than $10^{5} \mathrm{n} / \mathrm{s}$ because of the instability of glow plasma at $>30 \mathrm{kV}$ caused by overheating [5]. Further improvement in neutron yield by optimizing the discharge condition of the device is necessary for a quantitative 2D measurement.

As a preparation step for the neutronics experiment, this study aims to (i) calibrate the NIP using a radioactive neutron source with a known neutron production rate and (ii) optimize the discharge condition for efficient neutron generation. A ${ }^{252} \mathrm{Cf}$ spontaneous fission neutron source was selected because its average neutron energy $(2.3 \mathrm{MeV})$ is similar to that of DD fusion neutron $(2.45 \mathrm{MeV})$. A high voltage was applied for the discharge of the device due to its greater contribution to neutron yield than electric current [5]. Electric current was minimized to avoid the instability during an irradiation test.

\section{Method}

A neutron imaging plate (NIP) (Fuji-film BASND2025) composed of surface protective, photostimulated luminescence (PSL) material, ferrite, and support layers was used. The PSL material layer contained a fine mixture of PSL material $\left(\mathrm{BaBrF}: \mathrm{Eu}^{2+}\right)$ and $\mathrm{Gd}$ converter particles with a $\mathrm{Ba}$ Gd mixing ratio of 1:1. To minimize the fading effect where the recorded data on the imaging plate is gradually erased by visible light [9], the NIP was calibrated in a dark room. $\mathrm{A}{ }^{252} \mathrm{Cf}$ neutron source in a cylinder-shaped stainless container $(10 \mathrm{~mm} \phi, 10 \mathrm{mmh}$ ) was used for calibration of the NIP. The specific activity and neutron production rate of the source were $1.4 \times 10^{4} \mathrm{~Bq}$ and $1.6 \times 10^{3} \mathrm{n} / \mathrm{s}$, respectively. ${ }^{252} \mathrm{Cf}$ generates not only fission neutrons but also prompt $\gamma$ rays with an effective energy of $0.88 \pm 0.04 \mathrm{MeV}$ [10]. Lead is chosen as a shielding material because of its excellent absorption property of $\mathrm{X}$ - and $\gamma$-rays while maintaining good transparency for thermal neutron [11]. $\gamma$-rays were detected by a Geiger-Müller (GM) counter (TGS-121, Hitachi Aloka Medical).

Fig. 1 shows the calibration procedure for the NIP using the ${ }^{252} \mathrm{Cf}$ neutron source. The NIP was exposed to neutrons for various exposure times from $40 \mathrm{~min}$ to 165 $\mathrm{h}$ where prompt $\gamma$ rays were shielded by a lead filter. After an exposure, the NIP was immediately read out by a Storm 820 image reader from Molecular Dynamics using a beam of red-light laser (wavelength: $635 \mathrm{~nm}$ ). PSL density for one square centimeter around the center 
of a reading area $(10 \mathrm{~cm} \times 10 \mathrm{~cm}) P_{\mathrm{m}}\left(\mathrm{PSL} / \mathrm{cm}^{2}\right)$ was counted 10 times and then averaged. The pixel size in the reader was set to be $200 \mu \mathrm{m} \times 200 \mu \mathrm{m}$. The net PSL per area $P\left(\mathrm{PSL} / \mathrm{cm}^{2}\right)$ was obtained by the relation $P=$ $P_{\mathrm{m}}-P_{\mathrm{BG}}$ where $P_{\mathrm{BG}}\left(\mathrm{PSL} / \mathrm{cm}^{2}\right)$ is the background density of PSL. After the read out process, the NIP was reused after erasing the remaining data with a bright light in FLA image eraser (GE healthcare) for at least 60 min.

Simulations were performed by the Monte Carlo NParticle transport code MCNP-5.0 with the nuclear data from FENDL-2.1 and FENDL-3.1 library [12],[13]. The dosimetry cross section library IRDFF was employed for the Nat. $\mathrm{Gd}(\mathrm{n}, \gamma)$ reaction [14]. Neutron of the ${ }^{252} \mathrm{Cf}$ source was described by Watt fission energy spectrum [15]. Neutron transport in the calibration geometry was simulated with $10^{8}$ source particles by considering a 5 $\mathrm{cm}$ thick $\mathrm{Pb}$ filter, polyethylene block (thickness: 1-5 $\mathrm{cm}$ ), and the NIP. The distance between ${ }^{252} \mathrm{Cf}$ and the NIP was fixed to be $10 \mathrm{~cm}$. Neutron fluence on the read area of $1 \mathrm{~cm}^{2}$ square $\Phi\left(\mathrm{n} / \mathrm{cm}^{2}\right)$ is obtained by $\Phi=\phi \times t$ where $\phi$ and $t$ are neutron flux on the read area obtained by $\operatorname{MCNP}\left(\mathrm{n} / \mathrm{cm}^{2} / \mathrm{s}\right)$ and exposure time (s).

DD fusion neutrons were generated by a dischargetype cylindrical fusion device [5],[6]. The maximum current and voltage of the power supply were $30 \mathrm{~mA}$ and $125 \mathrm{kV}$ respectively. Both the anode and cathode of the device were coated with Ti to increase the neutron yield [6]. $\mathrm{D}_{2}$ gas pressure in the vacuum chamber was controlled to be $0.5-1.0 \mathrm{~Pa}$. The neutron yield was measured by GE Reuter Stokes ${ }^{3} \mathrm{He}$ gas filled detector calibrated with the ${ }^{252} \mathrm{Cf}$ neutron source placed at the center of the cathode in the device.

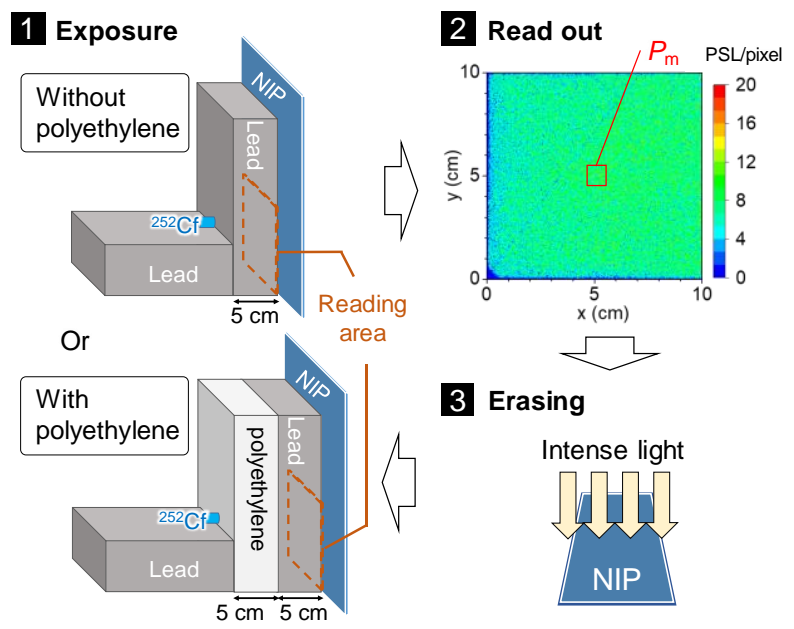

Fig. 1 Calibration procedure of the NIP using ${ }^{252} \mathrm{Cf}$.

\section{Results and Discussion}

\subsection{Calibration of NIP by ${ }^{252} \mathrm{Cf}$}

First, the shielding property of prompt $\gamma$-rays from ${ }^{252} \mathrm{Cf}$ source by a lead filter with thickness $x$ was assessed. Fig. 2 shows the normalized intensities of the $\gamma$-rays measured by the GM counter using a lead filter.
The thickness of lead filter $x$ was varied from 0 to $1.4 \mathrm{~cm}$ while keeping a distance of $5 \mathrm{~cm}$ between ${ }^{252} \mathrm{Cf}$ and the GM counter $5 \mathrm{~cm}$ (inset in Fig. 2). When the detector was in contact with the container of ${ }^{252} \mathrm{Cf}(x=0)$, the intensity was $10.4 \pm 1.5 \mathrm{count} / \mathrm{s}$; approximately 4 times higher than the background level. The attenuation coefficient of lead for prompt $\gamma$ rays from ${ }^{252} \mathrm{Cf}, \mu\left(\mathrm{cm}^{-1}\right)$, was obtained by fitting with $I / I_{0}=\exp (-\mu x)$ where $I$ and $I_{0}$ are $\gamma$-ray intensities (count/s) with and without a lead filter (thickness $x$ ), respectively. This fitting revealed $\mu$ to be $0.87 \pm 0.03 \mathrm{~cm}^{-1}$, indicating that a lead filter with 5 $\mathrm{cm}$ thickness reduces the intensity of the $\gamma$-ray to $1 / 100$. Although the NIP is as sensitive to the low energy $\gamma$-rays $(<300 \mathrm{keV})$ as to the thermal neutrons [11], the low energy $\gamma$-rays have high attenuation coefficients $(\mu>3.8$ $\mathrm{cm}^{-1}$ ) in lead and thus are readily removed by a lead filter with $5 \mathrm{~cm}$ thick. The NIP is 40 times less sensitive to the high energy $\gamma$-rays $(>300 \mathrm{keV})$, which could pass through the lead filter, than to the thermal neutrons [11]. Because of the adequate shielding property to $\gamma$-ray, a lead filter with a $5 \mathrm{~cm}$ thickness was chosen in the following experiments.

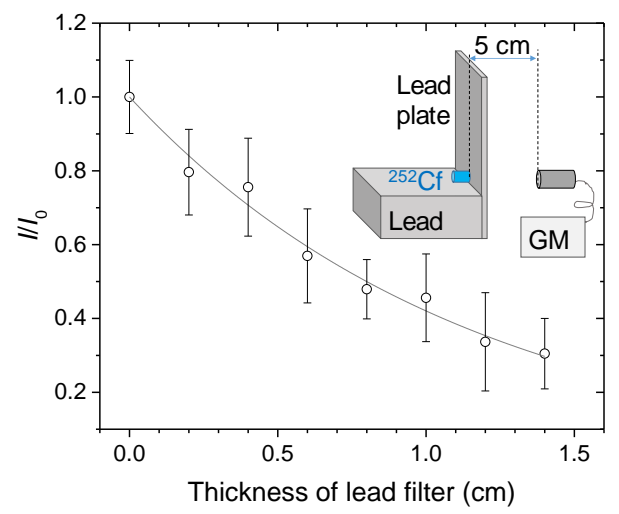

Fig. 2 Shielding property of a lead filter for prompt $\gamma$-rays from ${ }^{252} \mathrm{Cf}$ where inset represents geometry of the experiment.

By using the MCNP code, the influence of neutron moderation by polyethylene was investigated (data not shown). It was confirmed that the reaction rates of $\operatorname{Gd}(n, \gamma)$ in the NIP increased with the thickness of the polyethylene moderator placed between ${ }^{252} \mathrm{Cf}$ and the NIP. It is explained by $1 / v$ law for neutron capture by Gd in the NIP. The simulation result indicates that the sensitivity of the NIP can be improved by moderating neutron velocity. Therefore, two types of geometries were tested for the calibration; "without polyethylene" was a test only with the lead filter, while "with polyethylene" used a $5 \mathrm{~cm}$ thick polyethylene between ${ }^{252} \mathrm{Cf}$ and the lead filter. Fig. 3 shows the obtained relationships between the net PSL per area and $\Phi$ with and without the polyethylene moderator. Both results showed linear relationships, namely $P \propto \Phi^{k}$ ( $k$ : slope of line), in the range of neutron fluence between $10^{3}$ and $10^{7} \mathrm{n} / \mathrm{cm}^{2}$. The obtained slopes with and without polyethylene were $0.95 \pm 0.02$ and $0.96 \pm 0.05$, respectively. The obtained calibration lines were almost parallel to the previously reported relationship $P \propto \Phi^{0.973}$ 
in the neutron fluence from $10^{3}$ to $10^{8} \mathrm{n} / \mathrm{cm}^{2}$ measured in the Rikkyo TRIGA-II reactor [8]. The linearities are expected to be further maintained up to the order of $10^{9}$ $\mathrm{n} / \mathrm{cm}^{2}$ based on the previous report [8]. The sensitivity was improved with the polyethylene moderator, which was consistent with the simulation results. The higher the neutron fluence was, the lower the standard deviation of $P$ became (Fig. 3b). When a neutron fluence was larger than $10^{5} \mathrm{n} / \mathrm{cm}^{2}$, the standard deviation of $P$ was smaller than $5 \%$. Thus, a quantitative analysis of neutron fluence by the NIP requires $10^{6} \mathrm{n} / \mathrm{cm}^{2}$ of neutron fluence to be on the safe side.

(a)

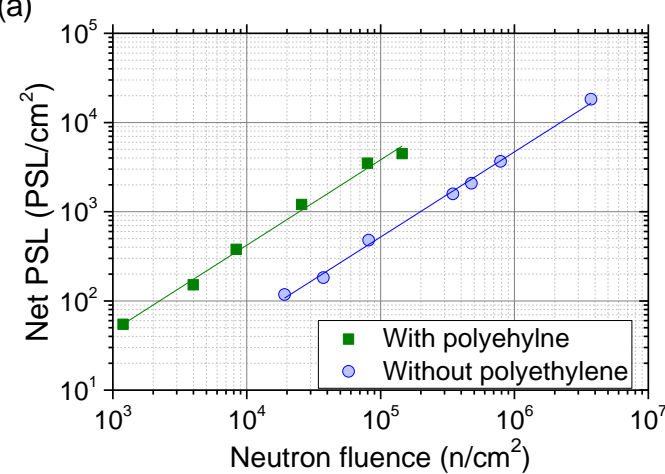

(b)

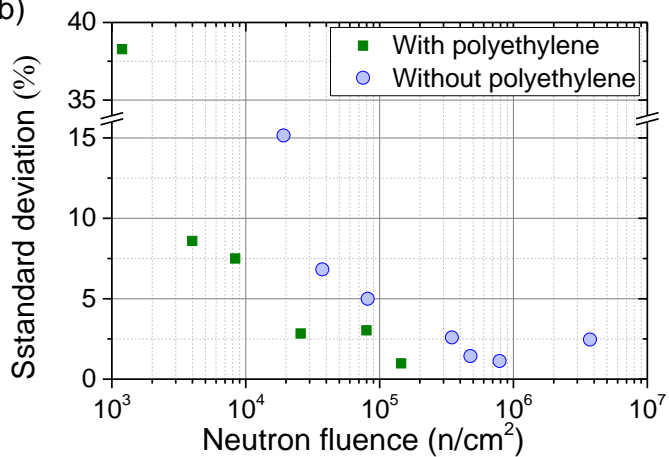

Fig. 3 Net PSL per area $\left(\mathrm{PSL} / \mathrm{cm}^{2}\right)$ and its standard deviation as a function of neutron fluence $\left(\mathrm{n} / \mathrm{cm}^{2}\right)$ obtained in the calibration using ${ }^{252} \mathrm{Cf}$.

\subsection{DD neutron irradiation}

Feasibility of the NIP as a quantitative detector using the DD fusion neutron device was assessed. The irradiation experiment system and geometry modeled for the MCNP simulations are shown in Fig.4. The NIP was placed $35 \mathrm{~cm}$ away from the neutron source and surrounded by lead blocks in order to shield X-rays from the fusion neutron device. A $5 \mathrm{~cm}$ thick polyethylene was placed between the NIP and the device for neutron moderation. The upper and lower parts of the NIP were shielded by lithium lead $(\mathrm{LiPb})$ for both $\mathrm{X}$-rays and neutrons. Returning neutrons were blocked by a boric acid solution and a polyethylene-boron composite placed behind and below the NIP. In Fig. 5, the neutron production rate from the device measured by the ${ }^{3} \mathrm{He}$ counter is plotted as a function of applied voltage and time during the DD neutron irradiation experiment. Applied voltage was increased up to $77 \mathrm{kV}$ by decreasing the $\mathrm{D}_{2}$ gas pressure in the vacuum chamber, while low currents were selected to keep electrical power smaller than approximately $300 \mathrm{~W}$ to avoid overheating of the device. Although a conditioning effect was observed at high voltages, the glow plasma was stable enough to count neutrons by the ${ }^{3} \mathrm{He}$ detector during the measurement time of $30 \mathrm{~s}$. The neutron yield increased exponentially with the applied voltage (Fig. 5a). A neutron production rate exceeding $10^{7} \mathrm{n} / \mathrm{s}$ was obtained when the applied voltage was higher than $75 \mathrm{kV}$. Fig. $5 \mathrm{~b}$ shows the irradiation history during the experiment with $4 \mathrm{~mA}$. The total neutron production during the experiment for $2.5 \mathrm{~h}$, corresponding to the gray-colored area in Fig. 5b, was estimated to be $6 \times 10^{10} \mathrm{n}$. By inputting the total neutron production value, neutron transport was simulated by MCNP with the modeled geometry (Fig. 4). The simulation showed that the neutron fluence in the NIP was $6 \times 10^{6} \mathrm{n} / \mathrm{cm}^{2}$. This result indicates that a neutron fluence higher than $10^{6} \mathrm{n} / \mathrm{cm}^{2}$ is expected in one irradiation hour. This satisfies the required neutron fluence for a quantitative analysis by the NIP where the standard deviation of $P$ is smaller than $5 \%$.
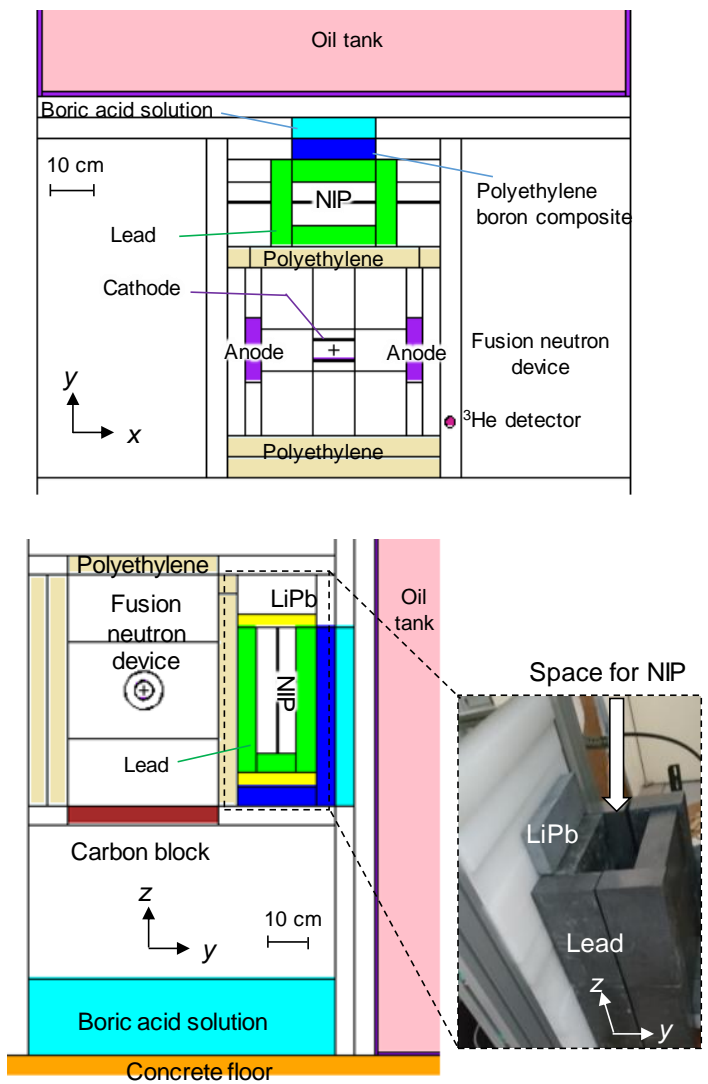

Fig. 4 Geometry of the neutron irradiation experiment and one modeled for the MCNP simulations.

Fig. 6 shows the simulated neutron spectrum in the NIP in the modeled geometry with a DD neutron source $(E=2.45 \mathrm{MeV})$. The neutron spectrum in the modeled system (black dashed line) was lower than the one in the calibration with the polyethylene moderator (blue dotted line). As the NIP has a high sensitivity to neutrons with 
lower energy, a correlation on neutron spectrum should be done, especially in energy region slower than fast neutron, by introducing correlation coefficient $K$. The correlated spectrum $K \phi_{\text {NIP }}$ with $K=2.5$ (red solid line in Fig. 6) agreed well with the spectrum in the calibration. Although a discrepancy was seen between fission and fusion neutrons, the calculation confirmed that contribution of fast neutrons $(>0.1 \mathrm{MeV})$ to the total $\operatorname{Gd}(\mathrm{n}, \gamma)$ reaction rate in the NIP is $0.1 \%$ and thus negligible. An experimental 2D measurement will be performed using the calibration curve and then compared with a 2D mesh of neutron flux distribution in MCNP simulations in a future work.

(a)
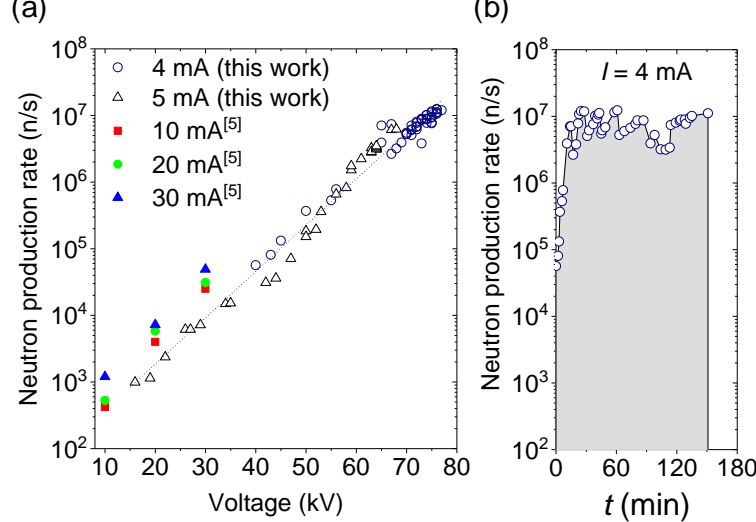

Fig. 5 Neutron production rate as functions of applied voltage (a) and experimental time (b) with the previous data [5]. Black dotted line in panel (a) is an exponential fitting curve.

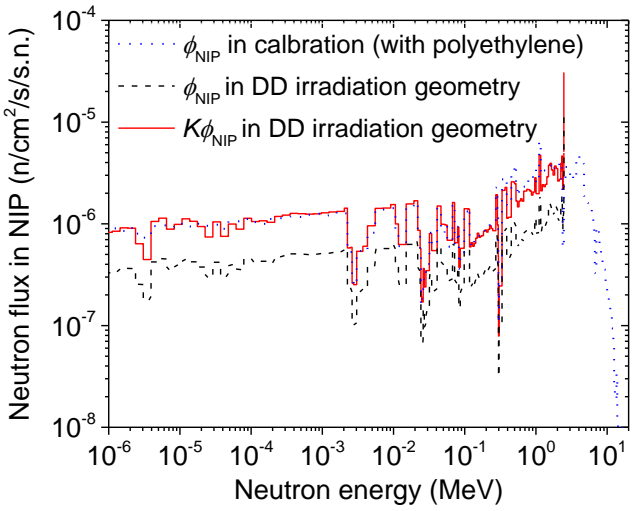

Fig. 6 Simulated neutron spectra in the NIP $\phi_{\text {NIP }}$ in the calibration with a $5 \mathrm{~cm}$ thick polyethylene, in the system with and without the correlation coefficient.

\section{Conclusion}

For a neutronics experiment, calibration of the neutron imaging plate (NIP) and optimization of the discharge condition of a fusion neutron device were carried out. The NIP was calibrated using the ${ }^{252} \mathrm{Cf}$ spontaneous fission neutron source with a $5 \mathrm{~cm}$ lead filter to remove $99 \%$ of prompt $\gamma$-rays from the source. The result showed a linear relationship between photostimulated luminescence and neutron fluence in the range of $10^{3}-10^{7} \mathrm{n} / \mathrm{cm}^{2}$ and an enhanced sensitivity by neutron moderation. A stable neutron generation by the device was successfully achieved with a low current and a high voltage up to $77 \mathrm{kV}$, resulting in neutron production rates higher than $10^{7} \mathrm{n} / \mathrm{s}$. The MCNP simulations showed that larger a neutron fluence greater than $10^{6} \mathrm{n} / \mathrm{cm}^{2}$ is obtained by an experiment for one irradiation hour. Consequently, the results showed that a quantitative two-dimensional measurement with the NIP is feasible with the fusion device by using the calibrated curves and applying a correlation factor on neutron energy. In future work, two-dimensional neutron measurement by the NIP will be performed and compared with MCNP simulations.

\section{Acknowledgments}

This work was supported by JSPS KAKENHI (grant number 17H06794). This work was performed with the support and under the auspices of the NIFS Collaborative Research Program (NIFS18KERF046).

\section{References}

[1] U. Fischer, R.L. Perel, H. Tsige-Tamirat, Fusion Eng. Des. 51 (2000) 761-768.

[2] P. Batistoni, M. Angelone, L. Bettinali, P. Carconi, U. Fischer, I. Kodeli, D. Leichtle, K. Ochiai, R. Perel, M. Pillon, I. Schäfer, Fusion Eng. Des. 82 (2007) 20952104.

[3] Y.K. Lee, Fusion Eng. Des. 85 (2010)1125-1128.

[4] Y. Verzilov, S. Sato, K. Ochiai, M. Wada, A. Klix, T. Nishitani, Fusion Eng. Des. 82 (2007) 1-9.

[5] K. Noborio, Y. Yamamoto, S. Konishi, J. Plasma Fusion Res. 9 (2014) 1306142.

[6] T. Kanagae, K. Noborio, Y. Yamamoto, S. Konishi, Proc. IEEE/NPSS $23^{\text {rd }}$ Symp. Fusion Eng. (2009). 10.1109/FUSION.2009.5226383.

[7] N. Niimura, Y. Karasawa, I. Tanaka, J. Miyahara, K. Takahashi, H. Saito, S. Koizumi, M. Hidaka, Nucl. Instrum. Methods Phys. Res. A 349 (1994) 521-525.

[8] H. Kobayashi, M. Satoh, Nucl. Instrum. Methods Phys. Res. A 424 (1999) 1-8.

[9] T. Bücherl, C. Rausch, H. von Seggern, Nucl. Instrum. Methods Phys. Res. A 333 (1993) 502-506.

[10] V.V. Verbinski, H. Weber, R.E. Sund, Phys. Rev. C 7 (1973) 1173.

[11] Y.K. Haga, S. Kumazawa, N. Niimura, J. Appl. Cryst. 32 (1999) 878-882

[12] The IAEA Nuclear Data Section, FENDL-2.1 Fusion Evaluated Nuclear Data Library, Vienna, Austria, INDC (NDS)-467, Version December 2004.

[13] Nuclear Data Libraries for Advanced Systems-Fusion Devices (FENDL 3.0), Summary Report of the Third Research Coordination Meeting IAEA, Vienna, Austria, 6-9 December, (2011).

[14] L.R. Greenwood, R. Paviotti-Corcuera, Summary Report of the Technical Meeting on International Reactor Dosimetry File: IRDF-2002, IAEA Report INDC (NDS)435, August 2002.

[15] A.B. Smith, P.R. Fields, J.H. Roberts, Phys. Rev. 108 (1957) 411. 